\title{
Persistent Heightened Cortisol Awakening Response and Adolescent Internalizing Symptoms: A 3-year Longitudinal Community Study
}

\author{
Stefanie A. Nelemans • William W. Hale III • Susan J. T. Branje • Pol A. C. van Lier • \\ Lucres M. C. Jansen • Evelien Platje • Tom Frijns • Hans M. Koot • Wim H. J. Meeus
}

Published online: 5 November 2013

(C) Springer Science+Business Media New York 2013

\begin{abstract}
An atypical Cortisol Awakening Response (CAR) has been related to adult anxiety and depression, but little is known about the association between long-term atypical CAR and adolescent anxiety and depression. This study aimed to longitudinally identify subgroups of adolescents with distinct levels of CAR (i.e., adolescents with and without persistent atypical (AR) and to examine their development of anxiety and depressive symptoms over 3 successive years. A community sample of 184 Dutch adolescents $\left(M_{\mathrm{age}}=14.99\right.$ at $\mathrm{T}_{1}, 57 \%$ boys) completed annual salivary cortisol assessments at home at time of awakening, and 30 and $60 \mathrm{~min}$ postawakening (i.e., CAR) for 3 successive years. Adolescents also reported annually on their anxiety and depressive disorder symptoms. Latent Class Growth Analysis suggested two subgroups of adolescents with respect to CAR: a "low" group with stable low levels of $\mathrm{AUC}_{\mathrm{g}}$ (Area Under the Curve with respect to the ground) over time and a "high" group with high and increasing levels of $\mathrm{AUC}_{\mathrm{g}}$ over time. Controlling for sex,
\end{abstract}

S. A. Nelemans $(\bowtie) \cdot$ W. W. Hale III $\cdot$ S. J. T. Branje $•$

W. H. J. Meeus

Research Centre Adolescent Development, Utrecht University, PO Box 80.140, 3508 TC Utrecht, The Netherlands

e-mail: s.a.nelemans@uu.nl

P. A. C. van Lier · H. M. Koot

Department of Developmental Psychology,

VU University Amsterdam, Amsterdam, The Netherlands

L. M. C. Jansen • E. Platje

Department of Child and Adolescent Psychiatry,

VU Medical Center, Amsterdam, Netherlands

T. Frijns

Trimbos Institute, Utrecht, The Netherlands

W. H. J. Meeus

Department of Developmental Psychology, Tilburg University, PO Box 90.153, 5000 LE Tilburg, The Netherlands the high and low CAR groups significantly differed in depressive symptoms only, but none of the anxiety disorder symptoms. More specifically, adolescents in the high CAR group showed significantly higher mean levels of depressive symptoms over time compared to adolescents in the low CAR group. These results suggest that persistent heightened CAR is a more consistent, yet modest, correlate of adolescent depressive symptoms than anxiety disorder symptoms.

Keywords Cortisol Awakening Response $(\mathrm{CAR}) \cdot$ Area under the curve with respect to the ground $\left(\mathrm{AUC}_{\mathrm{g}}\right) \cdot$ Anxiety .

Depression $\cdot$ Longitudinal $\cdot$ Adolescence

Anxiety and depression are among the most prevalent forms of psychopathology during adolescence (Merikangas et al. 2010). In addition to their high prevalence, a significant proportion of adolescent anxiety and depressive symptoms appear to have a chronic course (Bosquet and Egeland 2006; Holsen et al. 2000). Although there is overwhelming evidence that adolescent anxiety and depression are strongly related (Brady and Kendall 1992), several studies have argued that anxiety and depression also represent distinct entities (e.g., Hale et al. 2009). While our understanding of psychosocial and environmental risk factors for the development of adolescent anxiety and depressive symptoms is growing (Birmaher et al. 1996; Murray et al. 2009), our knowledge on neurobiological processes involved in these disorders, especially adolescent anxiety disorders, is still quite limited. It is also important to study these processes in adolescents from the general population as opposed to adolescents who have been diagnosed with a disorder, as has been done in many previous studies, because a potential referral bias may limit the generalizability of research findings in clinical samples (e.g., Hale et al. 2005). Therefore, this study aims to gain a better understanding of potential neurobiological factors involved in the development 
of adolescent anxiety and depressive disorder symptoms in the general population, by focusing on the association between these symptoms and secretory cortisol (i.e., stress) levels.

Cortisol is one of the major stress hormones secreted by the human physiological stress response system (including the Hypothalamic-Pituitary-Adrenal [HPA] axis) and is thereby involved in the regulation of many brain functions and behaviors, including those related to mood and emotions. Salivary measurements of the Cortisol Awakening Response (CAR; Pruessner et al. 1997) have been increasingly used as an important indicator of adrenal cortisol secretory activity. ${ }^{1} \mathrm{CAR}$ specifically refers to the marked morning rhythm normally exhibited by cortisol within the first hour after awakening, which is typically characterized by a rapid increase in levels upon awakening, peaking at around 30 min post-awakening, and declining thereafter (Wüst et al. 2000b). CAR has been found to be associated with several forms of adult mental health problems, including anxiety (Vreeburg et al. 2010b) and depression, with elevated CAR being one of the bestreplicated findings in the neurobiology of adult depression (Pruessner et al. 2003; Vreeburg et al. 2009).

Research on CAR and adolescent psychopathology is however fairly recent, hence it has not been particularly well studied or replicated yet. Only a few studies have focused on the association between CAR and adolescent anxiety and depression, despite of the knowledge that adolescence seems to be crucial developmental period for these disorders (Kessler et al. 2005). Of these few studies, most have focused on the association between CAR and adolescent depression. Results seem to resemble those found in adult studies, though less consistently, with heightened CAR being associated with heightened depressive symptoms (Guerry and Hastings 2011; Lopez-Duran et al. 2009). Of interest is that despite of the regular co-occurrence of depression and anxiety in adolescence, results of the few studies on the link between CAR and adolescent anxiety are highly inconsistent, with some studies suggesting a positive association between CAR and adolescent anxiety under specific conditions only (e.g., Greaves-Lord et al. 2007; Kallen et al. 2008).

One reason for the inconsistencies between these previous studies on the association between CAR and adolescent anxiety may be due to a focus on anxiety as a general and onedimensional construct rather than a focus on the multidimensional nature of anxiety, including a variety of

\footnotetext{
${ }^{1}$ It is noted, for example, by Spijker and Van Rossum (2012) that "it is complex to properly measure and assess the functioning of the HPA axis in humans" (p. 179). They also note that one of the most common approaches to evaluate HPA axis functioning is the measurement of basal cortisol levels in response to awakening, as a model for an endogenous stress response. Although CAR is more than simply a measure of HPA axis activity, we would refer the reader to Clow et al. (2010) and Fries et al. (2009) for a more detailed discussion of this issue, since this is beyond the scope of the present article.
}

heterogeneous symptoms. One commonly used approach of differentiating between different expressions of anxiety is by conceptualizing the multidimensional nature of anxiety in individual anxiety disorder symptoms as specified in the Diagnostic and Statistical Manual of the Mental Disorders (DSM-IV-TR; American Psychiatric Association 2000). Several theories (Warren and Sroufe 2004; Weems 2008) and empirical studies (Hale et al. 2008; Van Oort et al. 2009) have made important arguments for distinguishing between different anxiety disorder symptoms, not only in the clinical but also in the general population. Due to the unique characteristics of distinct anxiety disorder symptom dimensions and their unique relatedness to depressive symptoms, it is possible that some anxiety symptoms may show stronger associations with CAR than others. Indeed, Pêgo et al. (2010) have suggested that "patients with anxiety disorders show a wide spectrum of patterns of HPA activity, possibly because such disorders represent a very heterogeneous group of pathologies" ( $p$. 102). So while certain anxiety disorder symptoms may be expected to have a stronger association with CAR than others, studies that have directly compared the relationship between distinct adolescent anxiety and depressive disorder symptoms and CAR are almost nonexistent.

Another important limitation of previous studies on adolescent CAR is their cross-sectional nature, with CAR either being measured on one single day or measured across a few successive days. These studies thereby mask potentially important associations due to the limited time frame studied. Even more importantly, by taking an average of CAR across multiple days these studies have not considered potentially important individual differences in levels of CAR over time (i.e., intra-individual patterns of CAR over time). Indeed, research on the association between CAR and adolescent anxiety and depression has not assessed CAR annually across several years, nor focused specifically on the potential importance of individual differences in CAR (over time) in association with adolescent anxiety and depressive symptoms. Adolescents, however, do not only demonstrate large individual differences in CAR at any single point in time (Wüst et al. 2000b), but adolescents' CAR also shows only moderate to low rank-order stability over time (Platje et al. 2013). By not taking into account individual differences in CAR across time, previous studies have neglected to take into account possible stability (or change) in adolescents' CAR over time. While short-term releases of cortisol are adaptive and necessary for everyday functioning, prolonged releases of cortisol (e.g., indicated by persistent heightened levels of CAR over time) may be associated with negative adolescent mental health outcomes.

Indeed, Adam and Kumari (2009) suggest (based on research of Lupien et al. 1998) that chronically high levels of cortisol may be more important in relation to negative outcomes than just one time measurements of high cortisol. 
This emphasizes the need for a research approach that specifically focuses on intra-individual patterns of CAR over time and possible chronicity in adolescents' CAR curves, since such an approach may reveal important distinctions between subgroups of adolescents that may seem to have similar CAR patterns at any one measurement point. A subgroup of adolescents with persistent heightened CAR over a longer period of time may be especially likely to demonstrate heightened anxiety and depressive symptoms. Until now, no study has employed such a longitudinal approach to test how stability and chronicity in adolescents' CAR curves is associated with symptoms of anxiety and depression.

Therefore, the present study aimed to identify subgroups of adolescents with different patterns of CAR across 3 years, and to relate these different patterns of CAR to developmental trajectories of different anxiety disorder symptoms and depressive symptoms in adolescents from the general population. As previously noted, studies with adolescent samples from the general population are important because a potential referral bias limits the generalizability of research findings in clinical samples (e.g., Hale et al. 2005). We anticipated finding a subgroup of adolescents with persistent heightened CAR over 3 successive years (i.e., "high risk" developmental profile of CAR) in addition to a larger group of adolescents with more typical and lower CAR curves over 3 successive years (i.e., "low risk" developmental profile of CAR).

In line with previous studies and suggestions, we expected adolescents with persistent heightened CAR across 3 years to show higher levels of depressive symptoms over the studied period than adolescents with more typical and lower CAR curves. Furthermore, we explored potential differential associations between CAR and distinct anxiety disorder symptoms (Generalized Anxiety Disorder, Panic Disorder, Separation Anxiety Disorder, and Social Anxiety Disorder) by examining whether adolescents with persistent heightened CAR across 3 years also reported higher levels of distinct anxiety symptoms over the studied period than adolescents with more typical and lower CAR curves. Based on the unique characteristics of distinct anxiety disorder symptoms, we expected that some anxiety symptoms could have stronger associations with CAR than others. Pêgo et al. (2010) have indeed suggested that "anxiety disorders represent a heterogeneous group of disorders in which a variety of neurochemical systems may be involved..." (p. 103). No specific hypotheses could, however, be formulated in respect to which anxiety disorder symptoms would be stronger associated with CAR than others, because of the inconsistencies in previous studies and nonexistence of studies directly comparing different anxiety disorder symptoms in relation to (individual differences in long-term measured) CAR. Finally, because previous research has suggested important sex differences in
CAR (Pruessner et al. 1997; Wüst et al. 2000b) and mean levels of anxiety and depressive symptoms during adolescence (Cyranowski et al. 2000; McLean and Anderson 2009), we also examined the results of this study including sex as a covariate.

\section{Method}

\section{Participants}

Participants in this 3-year longitudinal community study were 184 adolescents ( $57 \%$ boys) with a mean age of 14.99 years ( $S D=0.42$, ranging from 14 to 16 years) at the start of the study. All adolescents identified themselves as being ethnic Dutch. Data for this study are part of the Research on Adolescent Development And Relationships (RADAR) project, a larger ongoing longitudinal community study on adolescent development and relationships. In the RADAR study, 437 adolescents $\left(56 \%\right.$ boys, $M_{\text {age }}=15.02, S D_{\text {age }}=$ $0.45)$ provided informed consent on the cortisol measurements, and 411 adolescents (56\% boys, $M_{\text {age }}=$ $\left.15.02, S D_{\text {age }}=0.44\right)$ provided at least one saliva sample over 3 years.

In the present study, we included only participants who completed all annual salivary cortisol measurements and relevant questionnaires over 3 successive years $(N=184)$. The study sample $(n=184)$ did not significantly differ from those who did not complete all salivary cortisol measurements and relevant questionnaires $(n=227)$ in terms of $\operatorname{sex}\left(\chi^{2}(1)=\right.$ $0.09, p=0.77$, Cramer's $V=0.02)$, age $(F(1,409)=1.23$, $\left.p=0.27, \eta^{2}=0.00\right)$, depressive symptoms $(F(1,406)=2.60$, $\left.p=0.11, \eta^{2}=0.01\right)$, and anxiety disorder symptoms $(F(4$, 403) $=1.13, p=0.34, \eta^{2}=0.01$ ).

\section{Procedure}

Participants were recruited from randomly selected (out of all regular primary education) schools in the western and central regions of the Netherlands. From all schools that were approached for this study, $69 \%$ were willing to participate. From these schools, adolescents of Dutch origin from twoparent families with a sibling $\geq 10$ years of age were randomly selected for participation. Before the start of the study, participants and their parents received a complete description of the study and $70 \%$ of the selected families $(N=497)$ provided active written informed consent to participate. The final RADAR sample contained only one adolescent (with some exceptions including two adolescents) from every school.

For each of the 3 successive years the participants were given a saliva sampling kit for home use to collect three morning cortisol samples (the exact procedure is explained 
into more detail under the section on the measurement of the Cortisol Awakening Response). In addition, the participants completed annual self-report questionnaires during a home visit. Adolescents received a small monetary compensation (€50, approximately US \$65) for every annual measurement they completed both the questionnaires and the cortisol samples. Of the 411 adolescents that provided both informed consent on the cortisol measurements and at least one saliva sample over 3 years, 184 adolescents completed all annual salivary cortisol measurements. This study was approved by the Board of the local research institute and by the Medical Ethical Committee of the Utrecht Medical Centre.

\section{Measures}

Anxiety Symptoms We used the Dutch version of the original 38-item Screen for Child Anxiety Related Emotional Disorders (SCARED; Birmaher et al. 1997; Hale et al. 2005) to assess adolescent anxiety disorder symptoms. The SCARED is a self-report questionnaire measuring four anxiety disorder symptom dimensions in children and adolescents that are directly related to the individual anxiety disorders in the DSM-IV-TR, namely symptoms of Generalized Anxiety Disorder (GAD), Panic Disorder (PD), Separation Anxiety Disorder (SepAD), and Social Anxiety Disorder (SAD).

Items were rated on a 3-point scale, ranging from 1 (almost never) to 3 (often). Sample items include "I am a worrier" (GAD), "When I am frightened, I feel like I am choking" (PD), "I don't like being away from my family" (SepAD), and "I don't like to be with people I don't know" (SAD). Internal consistency for the anxiety disorder symptom dimensions were found to be acceptable to good across waves, as the ranges of Cronbach's alpha for the four anxiety disorder symptom dimensions were between 0.88 and 0.90 for GAD, between 0.84 and 0.90 for PD, between 0.61 and 0.70 for SepAD, and between 0.83 and 0.86 for SAD, respectively. Psychometric properties of the SCARED have shown to be good in several previous studies, including good discriminant validity (i.e., differentiating between youths with and without anxiety disorders, between youth with distinct anxiety disorders, and between children with anxiety disorders and children with depressive disorders; Birmaher et al. 1997, 1999; Hale et al. 2005) and good convergent validity (i.e., SCARED showed strong sensitivity and specificity compared to A-DISC and was positively and meaningfully related to other anxiety questionnaires; Muris et al. 2000, 2002). In a meta-analysis, Hale et al. (2011) further concluded that the SCARED has robust psychometric properties and can be utilized as a screening instrument for DSM-IV-TR anxiety disorder symptoms. Furthermore, in a review of anxiety rating scales the SCARED was concluded to be one of the best self- report instruments to evaluate anxiety disorder symptoms (Myers and Winters 2002), making the SCARED a valid screening instrument with respect to the multidimensional nature of anxiety symptoms.

Depressive Symptoms To assess adolescent depressive symptoms, we used the Dutch version of Reynolds Adolescent Depression Scale - second edition (RADS-2; Reynolds 2000). The RADS- 2 consists of 23 items, measured on a 4-point scale ranging from 1 (almost never) to 4 (usually). Sample items include "I am sad" and "I feel like crying". Internal consistency for this scale was found to be good across waves, as Cronbach's alpha ranged from 0.94 to 0.95. Previous studies have shown good psychometric properties for the RADS- 2 in adolescence (Osman et al. 2010).

Cortisol Awakening Response (CAR) Adrenal cortisol secretory activity was measured annually by saliva sampling of the Cortisol Awakening Response (CAR; Pruessner et al. 1997; Wüst et al. 2000b). To assess CAR, saliva samples were obtained at three time points in the morning on a typical weekday during the school year; at awakening $\left(T_{1}\right)$, $30 \mathrm{~min}$ after awakening $\left(\mathrm{T}_{2}\right)$, and $60 \mathrm{~min}$ after awakening $\left(\mathrm{T}_{3}\right)$. Participants were instructed to collect their saliva through a small straw into a polypropylene tube, and label these tubes with the time and date of sampling. They were instructed not to eat, drink, smoke, or brush their teeth in the half-hour before sampling, to store the tubes with their saliva directly into the freezer after collection, and to send them back to the research center the same day. Adherence to the sampling protocol was assessed by self-report and by cross checking self-reported times of sampling.

After samples were returned to the research center, they were stored at $-20{ }^{\circ} \mathrm{C}$ until analysis. Salivary cortisol levels were analyzed using electrochemiluminescence immunoassay (E170 Roche, Switzerland). The lower detection limit was $0.5 \mathrm{nmol} / \mathrm{L}$, and the mean intra- and inter-assay coefficients of variation were $3 \%$ and $12 \%$, respectively, which represent acceptable coefficients of variation (Nicolson 2008; Salimetrics 2012). Exclusion criteria regarding the cortisol data were: incorrect sampling time, unclear how was sampled (not registered), or contaminated (e.g., by smoking or brushing teeth). Extreme cortisol values ( $>3$ SD from average) were also removed. For all adolescents with complete CAR data across the 3 years for this study $(n=184)$ we calculated the "Area Under the Curve with respect to the ground" $\left(\mathrm{AUC}_{\mathrm{g}}\right.$; Pruessner et al. 2003). CAR AUC $\mathrm{g}$ is considered a summary parameter of the repeated measurements of CAR $(0,30$, and $60 \mathrm{~min}$ after awakening), and, as such, is an estimate of total adrenal cortisol secretion during the first hour after awakening. 
Statistical Analyses

Latent Class Growth Analysis (LCGA; Reinecke 2006) in Mplus version 6.11 (Muthén and Muthén 2010) was used to identify subgroups of adolescents with different development of CAR AUC $\mathrm{g}_{\mathrm{g}}$ over time. Statistical techniques such as LCGA "have been increasingly recognized for their usefulness for identifying homogeneous subpopulations within the larger heterogeneous population and for the identification of meaningful groups or classes of individuals" (Jung and Wickrama 2008, p. 302). LCGA is a person-centered analysis technique, which examines the probability that large individual differences in a certain outcome measure can be captured within multiple more homogenous subgroups of individuals. LCGA makes it possible to look for naturally present subgroups of adolescents with different $\mathrm{CAR} \mathrm{AUC}_{\mathrm{g}}$ over time based on a number of fit criteria. More specifically, we used LCGA in this study to examine whether the large individual differences in adolescents' CAR AUC $\mathrm{g}_{\mathrm{g}}$ over 3 successive years could be captured within more homogenous subgroups of adolescents with different development of CAR $\mathrm{AUC}_{\mathrm{g}}$ over time.

We used four criteria to determine the number of groups with distinct development of CAR AUC $\mathrm{g}_{\mathrm{g}}$ over 3 successive years. First, we examined if adding an additional group would result in an improvement of model fit. A decrease in the Sample Size Adjusted Bayesian Information Criterion (SSA BIC) and a significant adjusted Lo, Mendell, and Rubin likelihood ratio test (adj. LMR-LRT) are indicative of this. Second, entropy - a standardized measure of classification quality - had to be acceptable. Entropy values range from 0 to 1 , with values of 0.75 or higher indicating acceptable classification accuracy (Reinecke 2006). Third, we evaluated the interpretability of the groups. If an additional group was found to be a slight variation of a group already found in a lower class solution, we chose the most parsimonious model. Finally, every group had to cover at least $5 \%$ of the sample for meaningful interpretation and further analysis.

This model selection procedure was used to determine the best-fitting LCGA model with adequate class sizes for subsequent analysis. Because previous research has suggested important sex differences in CAR (Pruessner et al. 1997; Wüst et al. 2000b), we examined potential sex differences in CAR $\mathrm{AUC}_{\mathrm{g}}$ within the final class model. We then conducted Repeated Measures MANOVA in SPSS to examine whether groups of adolescents with different development of CAR $\mathrm{AUC}_{\mathrm{g}}$ over time (which had resulted from LCGA) differed significantly in anxiety disorder symptoms and depressive symptoms over time (i.e., all these variables were analyzed in one Repeated Measures MANOVA). Because research has suggested important sex differences in levels of anxiety and depressive symptoms during adolescence (Cyranowski et al. 2000; McLean and Anderson 2009), we also examined the results with sex included as a covariate in the analyses.

\section{Results}

\section{Descriptive Statistics}

Table 1 provides an overview of the mean levels of all study variables over the studied time period of 3 successive years. Rank-order stability was low for the Cortisol Awakening Response "Area Under the Curve with respect to the ground" $\left(\mathrm{CAR} A \mathrm{AUC}_{\mathrm{g}}\right.$ ), with a correlation of 0.25 between successive waves. Hence there appeared to be large individual differences in the stability of CAR $\mathrm{AUC}_{\mathrm{g}}$ over time. Rank-order stability was higher for the different anxiety disorder symptoms $(>0.51)$ and depressive symptoms $(>0.68)$. Furthermore, within-wave correlations between the different anxiety disorder symptoms ranged from 0.32 to 0.64 at $\mathrm{T}_{1}$, from 0.39 to 0.59 at $\mathrm{T}_{2}$, and from 0.32 to 0.58 at $\mathrm{T}_{3}$, and correlations between the different anxiety disorder symptoms and depressive symptoms ranged from 0.28 to 0.74 at $T_{1}$, from 0.40 to 0.74 at $\mathrm{T}_{2}$, and from 0.37 to 0.75 at $\mathrm{T}_{3}$, with the highest correlations between GAD symptoms and depressive symptoms.

\section{Individual Differences in Cortisol Awakening Response} over 3 Successive Years

Based on the four criteria previously described, we decided upon a 2-class solution as the best fitting LCGA model (Entropy: 0.76, adj. LMR-LRT: $p<0.001$ ). Adding a third group to the model resulted in serious estimation problems and suggested a group containing only one adolescent $(<1 \%$ of the sample). Thus, individual differences in CAR $\mathrm{AUC}_{\mathrm{g}}$ over 3 successive years appeared to be best captured within two different subgroups of adolescents. Figure 1 graphically displays the mean growth curves of CAR $\mathrm{AUC}_{\mathrm{g}}$ over time for the two subgroups of adolescents in the final LCGA model.

Table 1 Mean levels and standard deviations of all study variables over 3 years

\begin{tabular}{|c|c|c|c|c|c|c|}
\hline \multirow[t]{2}{*}{ Variable } & \multicolumn{2}{|l|}{ Time 1} & \multicolumn{2}{|l|}{ Time 2} & \multicolumn{2}{|l|}{ Time 3} \\
\hline & $M$ & $S D$ & $M$ & $S D$ & $M$ & $S D$ \\
\hline $\mathrm{CAR} \mathrm{AUC}_{\mathrm{g}}$ & 1024.92 & 353.72 & 1019.46 & 376.86 & 1184.21 & 483.05 \\
\hline Depression & 1.51 & 0.49 & 1.58 & 0.53 & 1.58 & 0.52 \\
\hline GAD & 1.37 & 0.41 & 1.41 & 0.44 & 1.39 & 0.45 \\
\hline PD & 1.17 & 0.24 & 1.18 & 0.30 & 1.17 & 0.28 \\
\hline SepAD & 1.25 & 0.25 & 1.21 & 0.25 & 1.18 & 0.22 \\
\hline SAD & 1.52 & 0.53 & 1.48 & 0.53 & 1.49 & 0.5 \\
\hline
\end{tabular}

CAR AUC ${ }_{g}$ Cortisol Awakening Response Area Under the Curve with respect to the ground; $G A D$ Generalized Anxiety Disorder; $P D$ Panic Disorder; SepAD Separation Anxiety Disorder; $S A D$ Social Anxiety Disorder. Depression was measured on a scale from 1 to 4 . All anxiety disorder symptoms were measured on a scale from 1 to 3 


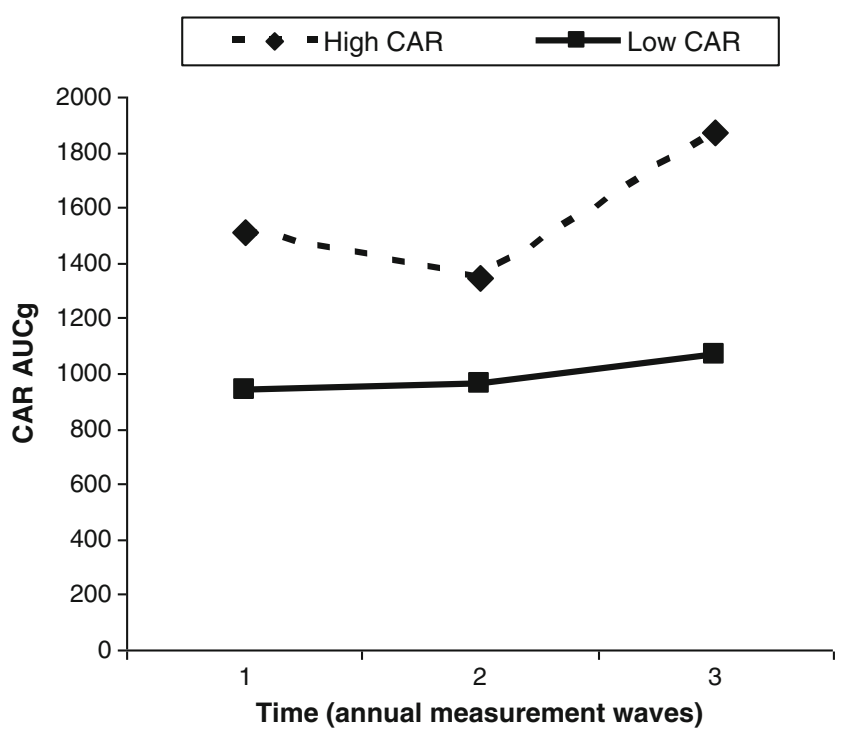

Fig. 1 Development of CAR AUC ${ }_{g}$ in the final LCGA model for the "high" and "low" CAR AUC $\mathrm{g}_{\mathrm{g}}$ groups. CAR AUC Cortisol Awakening Response Area Under the Curve with respect to the ground

The first class that was comprised of $85 \%$ of the adolescents $(n=157)$ was characterized by a low initial level of CAR $\operatorname{AUC}_{\mathrm{g}}(M=941.33)$ that remained fairly stable over time. The second class that contained the remaining $15 \%$ of the adolescents $(n=27)$ was characterized by a higher initial level of CAR AUC $\mathrm{AC}_{\mathrm{g}}(M=1510.99)$ that showed a non-linear increase over time. Based on the levels of CAR $\mathrm{AUC}_{\mathrm{g}}$ over time, we labeled the first group "low CAR" and the second group "high CAR".

The CAR groups did not significantly differ in time of waking/sampling $\left(F(3,180)=0.09, p=0.97, \eta^{2}=0.00\right)$ over the three annual measurement waves. In addition, sex did not significantly predict to which group adolescents were assigned $(p=0.85)$, nor did sex significantly predict the initial level of CAR AUC $\mathrm{A}_{\mathrm{g}}$ or the development of CAR $\mathrm{AUC}_{\mathrm{g}}$ over time within the two CAR groups $(p=0.20$ and $p=0.14$, respectively). We also found no significant sex differences in CAR AUC $_{\mathrm{g}}$ within both the low CAR group $(F(3,153)=0.14$, $\left.p=0.94, \eta^{2}=0.00\right)$ and the high CAR group $(F(3,23)=1.79$, $\left.p=0.18, \eta^{2}=0.19\right)$ over the three annual measurement waves.

Longitudinal Associations Between the Cortisol Awakening Response and Anxiety Disorder Symptoms and Depressive Symptoms

Most relevant to the aim of our study, the multivariate test in our unconditional Repeated Measures MANOVA suggested significant overall differences between the CAR groups (i.e., main effect of between-subjects factor) in anxiety and depressive symptoms $\left(F(5,178)=2.69, p=0.02, \eta^{2}=0.07\right)$, representing a medium effect size. More specifically, we found statistically significant differences between the two
CAR groups in depressive symptoms $(F(1,182)=5.01$, $p=0.01$ (one-tailed), $\eta^{2}=0.03$ ), Panic Disorder (PD) symptoms $\left(F(1,182)=3.37, p=0.03\right.$ (one-tailed), $\left.\eta^{2}=0.02\right)$, and Separation Anxiety Disorder (SepAD) symptoms $(F(1$, $182)=6.68, p=0.01$ (one-tailed), $\eta^{2}=0.04$ ) over 3 successive years (representing small or small-to-medium effect sizes). Adolescents in the high $\mathrm{CAR} \mathrm{AUC}_{\mathrm{g}}$ group reported significantly higher mean levels of these symptoms across time than adolescents in the low CAR $\mathrm{AUC}_{\mathrm{g}}$ group (see Figs. 2 and 3). No significant differences between the two CAR groups were found in Generalized Anxiety Disorder (GAD) symptoms ( $p=0.15$, one-tailed) or Social Anxiety Disorder (SAD) symptoms ( $p=0.27$, one-tailed). In addition, results suggested no significant change in any of the symptoms over time (i.e., main effect of within-subjects factor), nor differences between the CAR $\mathrm{AUC}_{\mathrm{g}}$ groups in their change of depressive or anxiety disorder symptoms over time (i.e., interaction effect of between- and within-subjects factors).

Including sex as a covariate in our Repeated Measures MANOVA changed results regarding differences between the $\mathrm{CAR} \mathrm{AUC}_{\mathrm{g}}$ groups (i.e., main effect of between-subjects factor) in anxiety disorder symptoms only. While adolescents in the high CAR $\mathrm{AUC}_{\mathrm{g}}$ group still reported significantly higher mean levels of depressive symptoms than adolescents in the low CAR AUC $\mathrm{g}_{\mathrm{g}}$ group $(F(1,181)=4.18, p=0.02$ (onetailed), $\eta^{2}=0.02$ ), results no longer suggested significant differences between the two CAR groups in PD symptoms ( $p=0.14$, one-tailed) and SepAD symptoms ( $p=0.07$, onetailed). The multivariate test further suggested significant overall differences between boys and girls in anxiety and depressive symptoms $\left(F(5,177)=4.45, p=0.001, \eta^{2}=0.11\right)$, representing a medium effect size. More specifically, we

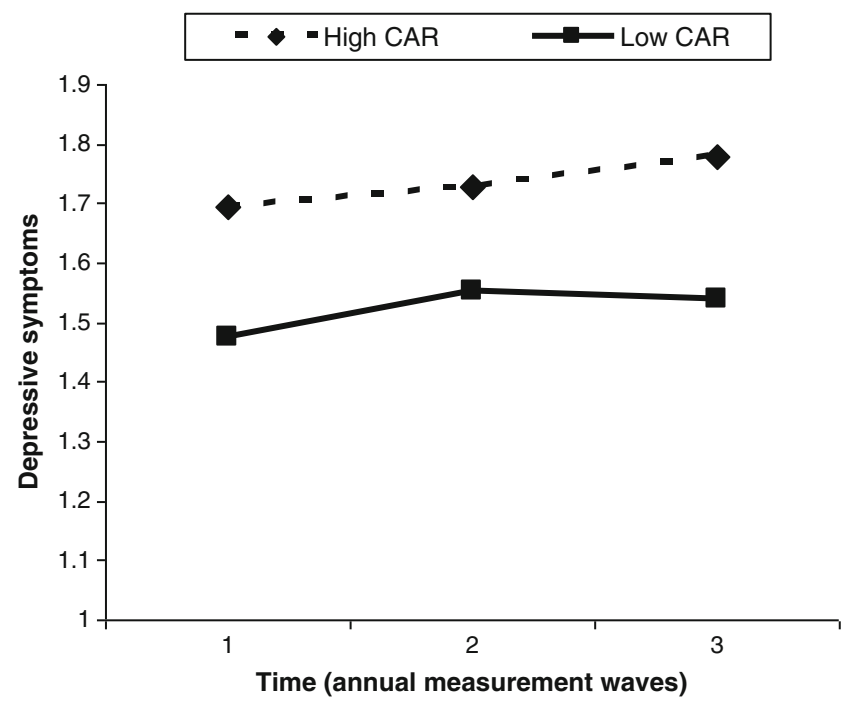

Fig. 2 Development of depressive symptoms for adolescents in the "high" and "low" CAR AUC groups. Note: The Y-axis displays item scores (1-4) 
Fig. 3 Development of Panic Disorder symptoms (left side) and Separation Anxiety Disorder symptoms (right side) for adolescents in the "high" and "low" CAR AUC groups. Note: The Y-axis displays item scores (1-3)

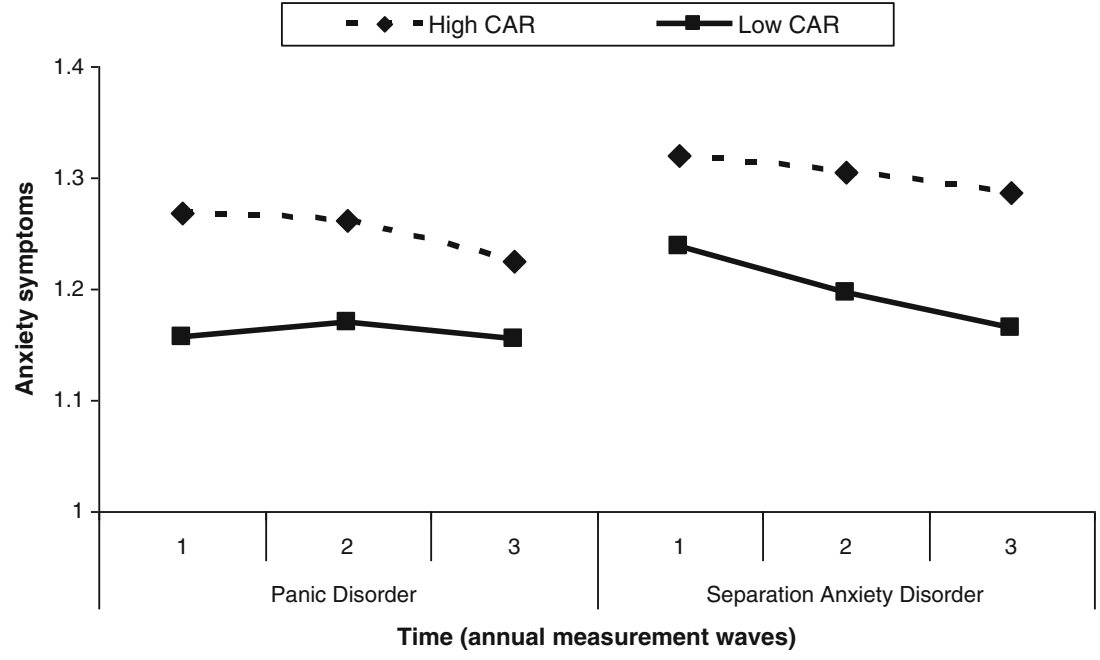

found significant sex differences in all anxiety disorder symptoms and depressive symptoms ( $p$ 's $\leq 0.001, \eta^{2}$ 's $=$ 0.06-0.08), with girls reporting significantly higher levels of anxiety and depression than boys.

\section{Discussion}

This 3-year longitudinal study examined the association between individual differences in stress levels (assessed by CAR) and symptoms of GAD, PD, SepAD, SAD, and depression in adolescents from the general population. This is thereby the first longitudinal study that distinguished between subgroups of adolescents from the general population based on persistence in patterns of CAR over 3 years and subsequently examined differences in their distinctive development of distinct anxiety and depressive disorder symptoms. Our results suggested that a small percentage of the adolescents showed persistent heightened CAR $\mathrm{AUC}_{\mathrm{g}}$ over 3 successive years, while the majority of adolescents showed stable lower CAR $\mathrm{AUC}_{\mathrm{g}}$ over time. In comparison to adolescents with stable low CAR $\mathrm{AUC}_{\mathrm{g}}$, adolescents with persistent heightened CAR $\mathrm{AUC}_{\mathrm{g}}$ showed slightly higher mean levels of depressive symptoms, but did not show higher mean levels of anxiety disorder symptoms when sex was accounted for. These results suggest that adolescents' persistent heightened levels of CAR $\mathrm{AUC}_{\mathrm{g}}$ are more strongly, yet modestly, related to depressive symptoms than to anxiety disorder symptoms, despite of the regular co-occurrence of adolescent depression and anxiety.

In line with previous studies on adults (Wüst et al. 2000b), we found large individual differences in adolescents' CAR. Moreover, we found only low rank-order stability of CAR $\mathrm{AUC}_{\mathrm{g}}$ (correlation of 0.25 between successive waves), suggesting large individual differences in the stability of adolescent stress levels over time. This finding demonstrates the importance of taking into account possible stability in adolescents' CAR curves. Furthermore, examining individual differences in trends of adolescent CAR AUC ${ }_{\mathrm{g}}$ levels over 3 successive years revealed important distinctions between subgroups of adolescents. In line with our expectations, we identified a small subgroup of adolescents $(15 \%$ of the total sample) with persistent heightened CAR $\mathrm{AUC}_{\mathrm{g}}$ over 3 successive years (see Fig. 1). These persistent heightened CAR $\mathrm{AUC}_{\mathrm{g}}$ levels reflect persistent adrenal cortisol secretion (i.e., stress levels) after awakening, and could be considered a stable marker for associated mental health problems. This was partially supported by our results on heightened levels of depressive symptoms for the adolescents with persistent heightened CAR AUC . .

Regarding adolescent depressive symptomatology, our results on the longitudinal association between persistent heightened CAR and adolescent depressive symptoms, which remained significant even after controlling for sex as a covariate, provide further evidence for the potentially important role of adrenal cortisol secretory activity in the neurobiology of adolescent depression. Although this study was not focused on the direction of the relationship between $\mathrm{CAR}$ and adolescent depressive symptoms, some studies have suggested that heightened CAR may actually be a biological trait vulnerability rather than a illness marker for depressive symptoms (Guerry and Hastings 2011; Vreeburg et al. 2010a). We should also state, however, that in the whole range of risk factors associated with adolescent anxiety and depressive symptoms the significance of CAR appears to be only modest, since the effect size of differences between the CAR groups in depressive symptoms was rather small. This finding is consistent with developmental psychopathology research and supports multifactor models of anxiety and depression (i.e., CAR is only one among many dynamic and interrelated factors associated with the development of anxiety and depressive symptoms). 
Regarding adolescent anxiety disorder symptomatology, our unconditional results suggested that adolescents with persistent heightened CAR reported significantly higher mean levels of PD and SepAD symptoms over time, but not GAD and SAD symptoms, than adolescents with stable lower CAR. However when sex was included as a covariate, differences between adolescents remained significant for depressive symptoms only, but no longer for PD and SepAD symptoms. These are interesting results for following reasons. First, the unconditional results may suggest that inconsistencies in previous studies on the association between CAR and adolescent anxiety may have been caused by not taking into account that adolescents may show large individual differences during adolescence with respect to stability and chronicity in CAR curves. Adolescents with persistent heightened CAR $\mathrm{AUC}_{\mathrm{g}}$ showed higher mean levels of specific anxiety disorder symptoms only. Secondly, the unconditional results suggest that despite of the strong association between anxiety and depressive symptoms, which seems to be especially true for GAD and depressive symptoms, chronically heightened cortisol levels are not uniformly associated with all of these internalizing symptoms. This result is consistent with results from other studies that suggest that although substantially related, anxiety (and specifically GAD) and depression are distinct psychopathologies (e.g., Hale et al. 2009). In addition, differences between adolescents with stable low CAR and adolescents with persistent heightened CAR remained significant for depressive symptoms only when sex was included as a covariate. These findings suggest that CAR, and thereby potentially certain aspects of the human physiological stress response system, may play a more prominent and consistent role in the development of depressive symptoms than anxiety disorder symptoms.

A related implication of these findings is that although persistent heightened CAR appeared to be specifically associated with higher levels of PD and SepAD symptoms and not with GAD and SAD symptoms, suggesting potential differences in stress activation may underlie different anxiety disorder symptoms, sex appeared to play a more important role in the development of all anxiety disorder symptoms than CAR. However, due to the relatively small sample size of the high risk CAR group, we cannot rule out that a lack of power might have influenced our results. In addition, even though our results suggested no significant sex differences in our CAR $\left(\mathrm{AUC}_{\mathrm{g}}\right)$ subgroups and thus no significant sex differences in intra-individual patterns of CAR over time, some studies have suggested that women show significantly higher mean levels of CAR or a significantly delayed decrease of cortisol after peak levels have been reached compared to men (e.g., Pruessner et al. 1997; Wüst et al. 2000b), resulting in higher CAR $\mathrm{AUC}_{\mathrm{g}}$ levels for women. Whether this may translate into adolescent girls having increased neuroendocrinological susceptibility to internalizing symptoms compared to boys and may be a partial explanation for the higher prevalence of anxious and depressive symptoms in adolescent girls compared to boys is yet to be further explored. However, sex differences in mean levels of CAR ( $\mathrm{AUC}_{\mathrm{g}}$ ) may not necessarily be reflected in sex differences in the associations between intraindividual patterns of CAR over a longer period of time and adolescent anxiety and depressive symptoms. Therefore, future studies should aim to include a larger sample size in their research designs to further examine potential sex differences in the role of stability and chronicity in adolescents' CAR curves (i.e., persistent heightened CAR) in developmental trajectories of adolescent anxiety and depressive disorder symptoms.

\section{Limitations and Future Research}

In addition to the aforementioned, our results should be considered in light of some limitations. First, caution should be given to the generalizability of our results to other adolescent populations, since this study employed a community sample of ethnic Dutch adolescents from twoparent families. Second, this study does not allow for any conclusions on direction of effects, since the longitudinal approach in this study was merely focused on associations of longitudinally measured CAR and adolescent anxiety disorder and depressive symptoms. Future research should further examine the potential predictive value of CAR in adolescent anxiety disorder and depressive symptoms and potential bidirectional relationships. Although our findings on concurrent associations over time make it difficult to speculate about potential implications of heightened CAR for future internalizing psychopathology, some studies have suggested that heightened CAR may be a biological trait vulnerability or a predictor of future psychopathology (Guerry and Hastings 2011; Vreeburg et al. 2010a).

In addition, while we have found that persistent heightened CAR is concurrently associated with higher depressive symptoms in the current study, some studies have found lower CAR to be associated with higher internalizing symptoms (e.g., Huber et al. 2006; Stetler and Miller 2005). These contradicting findings may relate to the severity of depression, with some studies investigating associations with CAR in Major Depressive Disorder (Huber et al. 2006; Stetler and Miller 2005) while others, including our study, are concerned with elevated depressed mood within the normal range (Pruessner et al. 2003). Studies have also suggested that nonlinear associations between the severity of anxiety and depressive symptoms and morning cortisol levels may exist (Evans et al. 2007; Veen et al. 2011; Wardenaar et al. 2011). In addition, the HPA axis is a self-regulating system that decreases its own activity through a negative feedback loop (i.e., down-regulation) to protect against prolonged exposure to elevated cortisol. It has been hypothesized that an initially 
hyperactive state in chronically stressed individuals might over time result in a hypoactive HPA axis with lower morning cortisol levels and thereby blunted CAR, due to downregulation of the HPA axis (Fries et al. 2005; Miller et al. 2007). Future longitudinal studies should take these potential contributors to the seemingly contradictory findings into account and may further want to explore this short term enhanced CAR versus potentially bunted CAR in the long term as well as potential nonlinear associations between anxiety and depression and morning cortisol levels.

Moreover, since biological and environmental factors interact with one another, future research could also examine how (persistent heightened) CAR might interact with important environmental influences to result in psychopathology, instead of only focusing on the direct association between CAR and adolescent symptoms of anxiety and depression. This sort of approach may be important, because previous studies have suggested that while main effects of (neuro)biological factors may be limited, biological factors may show important patterns of interaction with (social) environmental influences in association with psychopathology (e.g., with respect to geneenvironment interactions; Rutter et al. 2006). It should also be noted that although CAR has been shown to have a relatively strong genetic basis (e.g., Wüst et al. 2000a), studies have also shown that CAR appears to be influenced by psychological and situational factors such as work days versus weekend days, sleep quality, health behaviors such as smoking, and time of awaking (Adam and Kumari 2009; Fries et al. 2009; Pruessner et al. 1997; Wüst et al. 2000b). Future research may want to consider controlling more rigorously for potential confounders to get a more refined measure of neuroendocrine stress functioning, although results on potential external influences on CAR have been inconsistent across studies (Adam and Kumari 2009; Fries et al. 2009; Wüst et al. 2000b).

Furthermore, we acknowledge that a combination of CAR sampling across multiple days for multiple years would have been a better research procedure as our data is limited by single day measurements collected over three annual measurement occasions. Future research may try to include this more optimal sampling procedure, although a trade-off between feasibility (mostly related to financial costs and participant burden) likely has to be taken into account, as was the case in the current study. On a related note, we cannot ensure the validity of the sampling times in relation to the actual awakening time, which is a methodological challenge in the research field on CAR. Future research may want to use a more protocolled procedure of saliva sampling, though this may in turn impact the ecological validity of the samples (e.g., by having research assistants waking up participants for saliva sampling). Finally, sample size is another important issue is the kind of statistical modeling we used in this study (i.e., LCGA). With LCGA and comparable techniques, a larger sample size often results in more distinct groups of individuals. Future research involving a much larger sample size may want to explore whether different classes of adolescents with respect to CAR (e.g., including increasers and decreasers in CAR over time) can be identified and how these classes relate to adolescent mental health, including symptoms of depression and anxiety.

Altogether, to our knowledge the present study is one of the first to specifically focus on individual differences in adolescent adrenal cortisol secretory activity over a longer period of time (i.e., persistence and chronicity of adolescents' CAR curves) in association with symptoms of depression and distinct anxiety disorder symptoms. Clearly, our results require future replication. Although an increasing awareness is developing that cortisol (or more specifically the diurnal pattern of cortisol secretory activity, and especially CAR) has important influences on both physiological and psychological processes, individual differences in long-term CAR abnormalities have not yet received much attention in the literature. Because chronic abnormalities in CAR may have important conceptual implications (e.g., representing chronic stress), we believe that a longitudinal research focus on individual differences in adolescent CAR curves in association with mental health deserves more explicit attention in future research.

Acknowledgments Data of the RADAR study were used. RADAR has been financially supported by main grants from the Netherlands Organisation for Scientific Research (GB-MAGW 480-03-005, GBMAGW 480-08-006), and Stichting Achmea Slachtoffer en Samenleving (SASS), and various other grants from the Netherlands Organisation for Scientific Research, the VU University Amsterdam and Utrecht University. The authors would like to thank all schools and families for their participation.

\section{References}

Adam, E. K., \& Kumari, M. (2009). Assessing salivary cortisol in largescale, epidemiological research. Psychoneuroendocrinology, 34, $1423-1436$.

American Psychiatric Association. (2000). Diagnostic and statistical manual of mental disorders, 4th ed., Text revision (DSM-IV-TR). Washington, DC: American Psychiatric Association.

Birmaher, B., Ryan, N. D., Williamson, D. E., Brent, D. A., Kaufman, J., Dahl, R. E., et al. (1996). Childhood and adolescent depression: A review of the past 10 years. Part I. Journal of the American Academy of Child and Adolescent Psychiatry, 35, 1427-1439. doi:10.1097/ 00004583-199611000-00011.

Birmaher, B., Khetarpal, S., Brent, D., Cully, M., Balach, L., Kaufman, J., et al. (1997). The Screen for Child Anxiety Related Emotional Disorders (SCARED): Scale construction and psychometric characteristics. Journal of the American Academy of Child and Adolescent Psychiatry, 36, 545-553. doi:10.1097/00004583199704000-00018.

Birmaher, B., Brent, D., Chiappetta, L., Bridge, J., Monga, S., \& Baugher, M. (1999). Psychometric properties of the screen for child anxiety related emotional disorders (SCARED): A replication study. Journal of the American Academy of Child and Adolescent Psychiatry, 38, 1230-1236. doi:10.1097/00004583-199910000-00011.

Bosquet, M., \& Egeland, B. (2006). The development and maintenance of anxiety symptoms from infancy through adolescence in a 
longitudinal sample. Development and Psychopathology, 18, 517550. doi:10.1017/S0954579406060275.

Brady, E. U., \& Kendall, P. C. (1992). Comorbidity of anxiety and depression in children and adolescents. Psychological Bulletin, 111, 244-255. doi:10.1037/0033-2909.111.2.244.

Clow, A., Hucklebridge, F., Stalder, T., Evans, P., \& Thorn, L. (2010). The cortisol awakening response: More than a measure of HPA axis function. Neuroscience and Biobehavioral Reviews, 35, 97-103. doi:10.1016/j.neubiorev.2009.12.011.

Cyranowski, J. M., Frank, E., Young, E., \& Shear, M. K. (2000). Adolescent onset of the gender difference in lifetime rates of major depression. A theoretical model. Archives of General Psychiatry, $57,21-27$.

Evans, P., Forte, D., Jacobs, C., Fredhoi, C., Aitchison, E., Hucklebridge, F., et al. (2007). Cortisol secretory activity in older people in relation to positive and negative well-being. Psychoneuroendocrinology, 32 , 922-930. doi:10.1016/j.psyneuen.2007.06.017.

Fries, E., Hesse, J., Hellhammer, J., \& Hellhammer, D. H. (2005). A new view on hypocortisolism. Psychoneuroendocrinology, 30, 1010 1016. doi:10.1016/j.psyneuen.2005.04.006.

Fries, E., Dettenborn, L., \& Kirschbaum, C. (2009). The cortisol awakening response (CAR): Facts and future directions. International Journal of Psychophysiology, 72, 67-73. doi:10. 1016/j.ijpsycho.2008.03.014.

Greaves-Lord, K., Ferdinand, R. F., Oldehinkel, A. J., Sondeijker, F. E. P. L., Ormel, J., \& Verhulst, F. C. (2007). Higher cortisol awakening response in young adolescents with persistent anxiety problems. Acta Psychiatrica Scandinavica, 116, 137-144. doi:10.1111/j. 1600-0447.2007.01001.x.

Guerry, J. D., \& Hastings, P. D. (2011). In search of HPA axis dysregulation in child and adolescent depression. Clinical Child and Family Psychology Review, 14, 135-160. doi:10.1007/s10567-011-0084-5.

Hale, W. W., III, Raaijmakers, Q. A. W., Muris, P., \& Meeus, W. H. J. (2005). Psychometric properties of the Screen for Child Anxiety Related Emotional Disorders (SCARED) in the general adolescent population. Journal of the American Academy of Child and Adolescent Psychiatry, 44, 283-290. doi:10.1097/00004583200503000-00013.

Hale, W. W., III, Raaijmakers, Q. A. W., Muris, P., Van Hoof, A., \& Meeus, W. H. J. (2008). Developmental trajectories of adolescent anxiety disorder symptoms: A 5-year prospective community study. Journal of the American Academy of Child and Adolescent Psychiatry, 47, 556-564. doi:10.1097/CHI.0b013e3181676583.

Hale, W. W., III, Raaijmakers, Q. A. W., Muris, P., Van Hoof, A., \& Meeus, W. H. J. (2009). One factor or two parallel processes? Comorbidity and development of adolescent anxiety and depressive disorder symptoms. Journal of Child Psychology and Psychiatry, 50, 1218-1226. doi:10.1111/j.1469-7610.2009.02115.x.

Hale, W. W., III, Crocetti, E., Raaijmakers, Q. A. W., \& Meeus, W. H. J. (2011). A meta-analysis of the cross-cultural psychometric properties of the Screen for Child Anxiety Related Emotional Disorders (SCARED). Journal of Child Psychology and Psychiatry, 52, 80-90. doi:10.1111/j.14697610.2010.02285.x.

Holsen, I., Kraft, P., \& Vittersø, J. (2000). Stability in depressed mood in adolescence: Results from a 6-year longitudinal panel study. Journal of Youth and Adolescence, 29, 61-78.

Huber, T. J., Issa, K., Schik, G., \& Wolf, O. T. (2006). The cortisol awakening response is blunted in psychotherapy inpatients suffering from depression. Psychoneuroendocrinology, 31, 900-904. doi:10. 1016/j.psyneuen.2006.03.005.

Jung, T., \& Wickrama, K. A. S. (2008). An introduction to latent class growth analysis and growth mixture modeling. Social and Personality Psychology Compass, 2, 302-317. doi:10.1111/j. 1751-9004.2007.00054.x.

Kallen, V. L., Tulen, J. H. M., Utens, E. M. W. J., Treffers, P. D. A., De Jong, F. H., \& Ferdinand, R. F. (2008). Associations between HPA axis functioning and level of anxiety in children and adolescents with an anxiety disorder. Depression and Anxiety, 25, 131-141. doi: 10.1002/da.20287.

Kessler, R. C., Berglund, P., Demler, O., Jin, R., Merikangas, K. R., \& Walters, E. E. (2005). Lifetime prevalence and age-of-onset distributions of DSM-IV disorders in the national comorbidity survey replication. Archives of General Psychiatry, 62, 593-602. doi:10.1001/archpsyc.62.6.593.

Lopez-Duran, N. L., Kovacs, M., \& George, C. J. (2009). Hypothalamicpituitary-adrenal axis dysregulation in depressed children and adolescents: A meta-analysis. Psychoneuroendocrinology, 34, 1272-1283. doi:10.1016/j.psyneuen.2009.03.016.

Lupien, S. J., De Leon, M., De Santi, S., Convit, A., Tarshish, C., Nair, N. P. V., et al. (1998). Cortisol levels during human aging predict hippocampal atrophy and memory deficits. Nature Neuroscience, 1, 69-73. doi:10.1038/271.

McLean, C. P., \& Anderson, E. R. (2009). Brave men and timid women? A review of the gender differences in fear and anxiety. Clinical Psychology Review, 29, 496-505. doi:10.1016/j.cpr.2009.05.003.

Merikangas, K. R., He, J.-P., Burstein, M., Swanson, S. A., Avenevoli, S., Cui, L., et al. (2010). Lifetime prevalence of mental disorders in U.S. adolescents: Results from the national comorbidity survey replication-adolescent supplement (NCS-A). Journal of the American Academy of Child and Adolescent Psychiatry, 49, 980 989. doi:10.1016/j.jaac.2010.05.017.

Miller, G. E., Chen, E., \& Zhou, E. S. (2007). If it goes up, must it come down? Chronic stress and the hypothalamic-pituitary-adrenocortical axis in humans. Psychological Bulletin, 133, 25-45. doi:10.1037/ 0033-2909.133.1.25.

Muris, P., Merckelbach, H., Mayer, B., \& Prins, E. (2000). How serious are common childhood fears? Behaviour Research and Therapy, 38 , 217-228. doi:10.1016/S0005-7967(98)00204-6.

Muris, P., Merckelbach, H., Ollendick, T., King, N., \& Bogie, N. (2002). Three traditional and three new childhood anxiety questionnaires: Their reliability and validity in a normal adolescent sample. Behaviour Research and Therapy, 40, 753-772. doi:10.1016/ S0005-7967(01)00056-0.

Murray, L., Creswell, C., \& Cooper, P. J. (2009). The development of anxiety disorders in childhood: An integrative review. Psychological Medicine, 39, 1413-1423. doi:10.1017/S0033291709005157.

Muthén, L. K., \& Muthén, B. O. (2010). Mplus user's guide (6th ed.). Los Angeles: Muthén \& Muthén.

Myers, K., \& Winters, N. C. (2002). Ten-year review of rating scales. II: Scales for internalizing disorders. Journal of the American Academy of Child and Adolescent Psychiatry, 41, 634-659. doi:10.1097/ 00004583-200206000-00004.

Nicolson, N. A. (2008). Measurement of cortisol. In L. J. Luecken \& L. C. Gallo (Eds.), Handbook of physiological research methods in health psychology (pp. 37-74). Thousand Oaks: Sage Publications, Inc.

Osman, A., Gutierrez, P. M., Bagge, C. L., Fang, Q., \& Emmerich, A. (2010). Reynolds Adolescent Depression Scale-second edition: A reliable and useful instrument. Journal of Clinical Psychology, 66, 1324-1345. doi:10.1002/jclp.20727.

Pêgo, J. M., Sousa, J. C., Almeida, O. F. X., \& Sousa, N. (2010). Stress and the neuroendocrinology of anxiety disorders. Current Topics in Behavioral Neurosciences, 2, 97-118. doi:10.1007/7854_2009_13.

Platje, E., Vermeiren, R. R. J. M., Branje, S. J. T., Doreleijers, T. A. H., Meeus, W. H. J., Koot, H. M., et al. (2013). Long-term stability of the cortisol awakening response over adolescence. Psychoneuroendocrinology, 38, 271-280. doi:10.1016/j.psyneuen. 2012.06.007.

Pruessner, J. C., Wolf, O. T., Hellhammer, D. H., Buske-Kirschbaum, A., Von Auer, K., Jobst, S., et al. (1997). Free cortisol levels after awakening: A reliable biological marker for the assessment of adrenocortical activity. Life Sciences, 61, 2539-2549. doi:10.1016/ S0024-3205(97)01008-4. 
Pruessner, M., Hellhammer, D. H., Pruessner, J. C., \& Lupien, S. J. (2003). Self-reported depressive symptoms and stress levels in healthy young men: Associations with the cortisol response to awakening. Psychosomatic Medicine, 65, 92-99. doi:10.1097/01. PSY.0000040950.22044.10.

Reinecke, J. (2006). Longitudinal analysis of adolescents' deviant and delinquent behavior: Applications of latent class growth curves and growth mixture models. Methodology, 2, 100-112. doi:10.1027/ 1614-2241.2.3.100.

Reynolds, W. M. (2000). Reynolds adolescent depression scale - 2nd edition (RADS-2). Professional manual. Lutz: Psychological Assessment Resources.

Rutter, M., Moffitt, T. E., \& Caspi, A. (2006). Gene-environment interplay and psychopathology: Multiple varieties but real effects. Journal of Child Psychology and Psychiatry, 47, 226-261. doi:10. 1111/j.1469-7610.2005.01557.x.

Salimetrics (2012). Inter- and intra-assay coefficients of variability. Retrieved from http://www.salimetrics.com/spit-tips/

Spijker, A. T., \& Van Rossum, E. F. C. (2012). Glucocorticoid sensitivity in mood disorders. Neuroendocrinology, 95, 179-186. doi:10.1159/ 000329846.

Stetler, C., \& Miller, G. E. (2005). Blunted cortisol response to awakening in mild to moderate depression: Regulatory influences of sleep patterns and social contacts. Journal of Abnormal Psychology, 114, 697-705. doi:10.1037/0021-843X.114.4.697.

Van Oort, F. V. A., Greaves-Lord, K., Verhulst, F. C., Ormel, J., \& Huizink, A. C. (2009). The developmental course of anxiety symptoms during adolescence: The TRAILS study. Journal of Child Psychology and Psychiatry, 50, 1209-1217. doi:10.1111/j. 1469-7610.2009.02092.x.

Veen, G., Van Vliet, I. M., DeRijk, R. H., Giltay, E. J., Van Pelt, J., \& Zitman, F. G. (2011). Basal cortisol levels in relation to dimensions and DSM-IV categories of depression and anxiety. Psychiatry Research, 185, 121-128. doi:10.1016/j.psychres.2009.07.013.
Vreeburg, S. A., Hoogendijk, W. J. G., Van Pelt, J., DeRijk, R. H., Verhagen, J. C. M., Van Dyck, R., et al. (2009). Major depressive disorder and hypothalamic-pituitary-adrenal axis activity: Results from a large cohort study. Archives of General Psychiatry, 66, 617-626. doi:10.1001/archgenpsychiatry.2009.50.

Vreeburg, S. A., Hartman, C. A., Hoogendijk, W. J. G., Van Dyck, R., Zitman, F. G., Ormel, J., et al. (2010a). Parental history of depression or anxiety and the cortisol awakening response. British Journal of Psychiatry, 197, 180-185. doi:10.1192/bjp.bp.109. 076869.

Vreeburg, S. A., Zitman, F. G., Van Pelt, J., DeRijk, R. H., Verhagen, J. C. M., Van Dyck, R., et al. (2010b). Salivary cortisol levels in persons with and without different anxiety disorders. Psychosomatic Medicine, 72, 340-347. doi:10.1097/PSY.0b013e3181d2f0c8.

Wardenaar, K. J., Vreeburg, S. A., Van Veen, T., Giltay, E. J., Veen, G., Penninx, B. W. J. H., et al. (2011). Dimensions of depression and anxiety and the hypothalamo-pituitary-adrenal axis. Biological Psychiatry, 69, 366-373. doi:10.1016/j.biopsych.2010.09.005.

Warren, S. L., \& Sroufe, L. A. (2004). Developmental issues. In T. H. Ollendick \& J. S. March (Eds.), Phobic and anxiety disorders in children and adolescents: A clinician's guide to effective psychosocial and pharmacological interventions (pp. 92-115). New York: Oxford University Press.

Weems, C. F. (2008). Developmental trajectories of childhood anxiety: Identifying continuity and change in anxious emotion. Developmental Review, 28, 488-502. doi:10.1016/j.dr.2008.01. 001.

Wüst, S., Federenko, I., Hellhammer, D. H., \& Kirschbaum, C. (2000a). Genetic factors, perceived chronic stress, and the free cortisol response to awakening. Psychoneuroendocrinology, 25, 707-720. doi:10.1016/S0306-4530(00)00021-4.

Wüst, S., Wolf, J., Hellhammer, D. H., Federenko, I., Schommer, N., \& Kirschbaum, C. (2000b). The cortisol awakening response - normal values and confounds. Noise and Health, 2(7), 79-88. 